\title{
A Realtime Pattern Generator for Biped Walking
}

\author{
Shuuji Kajita, Fumio Kanehiro, Kenji Kaneko, Kiyoshi Fujiwara, \\ Kazuhito Yokoi and Hirohisa Hirukawa \\ Intelligent Systems Institute, \\ National Institute of Advanced Industrial Science and Technology (AIST) \\ AIST Tsukuba Central 2, Tsukuba, 305-8568 Japan \\ E-mail: s.kajita@aist.go.jp
}

\begin{abstract}
For realtime walking control of a biped robot, we analyze the dynamics of a three-dimensional inverted pendulum whose motions are constrained onto an arbitrarily defined plane. This analysis leads us a simple linear dynamics, the Three-Dimensional Linear Inverted Pendulum Mode (3D-LIPM). Geometric nature of trajectories under the 3D-LIPM is discussed, and an algorithm for walking pattern generation is presented. Experimental results of realtime walking control of a 12 d.o.f. biped robot HRP-2L using an input device such as a game pad are also shown.
\end{abstract}

\section{Introduction}

Research on humanoid robots and biped locomotion is currently one of the most exciting topics in the field of robotics and there exist many ongoing projects. Although some of those works have already demonstrated very reliable dynamic biped walking $[3,10,14]$, we believe it is still important to understand the theoretical background of biped locomotion.

A lot of researches dedicated to the biped walking pattern generation can be classified into two categories. The first group uses precise knowledge of dynamic parameter of a robot e.g. mass, location of center of mass and inertia of each link to prepare walking patterns. Therefore, it mainly relies on the accuracy of the model data $[3,5,10,14]$.

On the contrary, the second group uses limited knowledge of dynamics e.g. location of total center of mass, total angular momentum, etc. Since the controller knows little about the system structure, this approach much relies on a feedback control $[1,6,9,12,13,15]$.
In this paper, we take the standpoint of the second approach, and introduce a new modeling which represents the dynamics of a robot with limited parameters. The modeling, the Three-Dimensional Linear Inverted Pendulum Mode (3D-LIPM) is derived from a general three-dimensional inverted pendulum whose motion is constrained onto an arbitrarily defined plane [7].

It allows a separate controller design for the sagittal $(\mathrm{x}-\mathrm{z})$ and the lateral $(\mathrm{y}-\mathrm{z})$ motions and simplifies a walking pattern generation a great deal. This merit makes realtime walk generation possible.

The rest of the paper is organized as follows. Section 2 presents a nature of 3D Linear Inverted Pendulum Mode. Section 3 describes the details of 3D walking pattern generation. Section 4 shows experimental results. We conclude in Section 5 .

\section{Derivation of 3D Linear Inverted Pendulum Mode}

\subsection{Motion equation of a 3D inverted pendulum}

When a biped robot is supporting its body on one leg, its dominant dynamics can be represented by a single inverted pendulum which connects the supporting foot and the center of mass of the whole robot. Figure 1 depicts such an inverted pendulum consisting of a point mass and a massless telescopic leg. The position of the point mass $\boldsymbol{p}=(x, y, z)$ is uniquely specified by a set of state variables $\boldsymbol{q}=\left(\theta_{r}, \theta_{p}, r\right)$ where $\theta_{r}$ indicates the angle between the pendulum and the XZ-plane and $\theta_{p}$ indicates the angle between the pendulum and the XZ-plane (Fig. 1). The signs of these angles are defined to fit the right handed coordinate system, so we have $\theta_{r}<0$ and $\theta_{p}>0$ in the configuretaion of Fig. 1. $r$ indicates the length of massless 


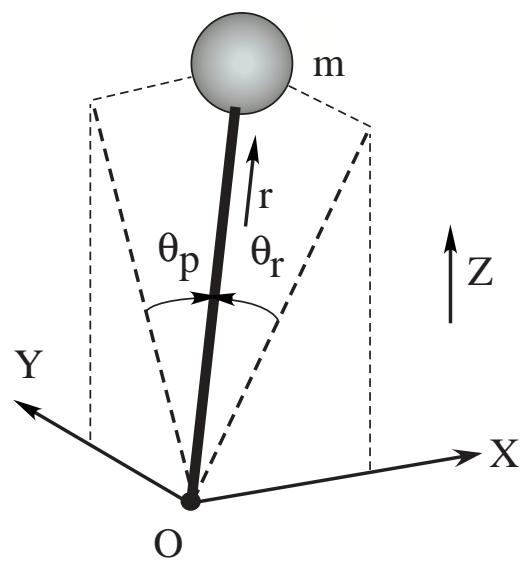

Figure 1: 3D Pendulum

leg which is the distance between the origin and the point mass.

Let $\left(\tau_{r}, \tau_{p}, f\right)$ be the actuator torque and force associated with the state variables $\left(\theta_{r}, \theta_{p}, r\right)$. With these inputs, the equations of motion of the $3 \mathrm{D}$ inverted pendulum in Cartesian coordinates are given by

$$
\begin{gathered}
m(-z \ddot{y}+y \ddot{z})=\frac{D}{C_{r}} \tau_{r}-m g y, \\
m(z \ddot{x}-x \ddot{z})=\frac{D}{C_{p}} \tau_{p}+m g x,
\end{gathered}
$$

where

$$
C_{r} \equiv \cos \theta_{r}, C_{p} \equiv \cos \theta_{p}, D \equiv \sqrt{{C_{r}{ }^{2}+C_{p}{ }^{2}-1}_{1}}
$$

$m$ is the mass of the pendulum, and $g$ is gravity acceleration.

\subsection{D Linear Inverted Pendulum Mode}

Although the moving pattern of the pendulum has vast possibilities, we want to select a class of motions that would be suitable for walking. For this reason, we apply constraints to limit the motion of the pendulum. The first constraint limits the motion in the plane with given normal vector $\left(k_{x}, k_{y},-1\right)$ and $\mathrm{z}$ intersection $z_{c}$,

$$
z=k_{x} x+k_{y} y+z_{c} .
$$

For a robot walking on a rugged terrain, the normal vector should match the slope of the ground and the $\mathrm{z}$ intersection should be the expected average distance of the center of the robot's mass from the ground. For further calculation, we prepare the second derivatives of Eq. (3),

$$
\ddot{z}=k_{x} \ddot{x}+k_{y} \ddot{y} .
$$

Substituting these constraints into Eqs. (1) and (2), we obtain the dynamics of the pendulum under the constraints. From straightforward calculations, we get

$$
\begin{aligned}
& \ddot{y}=\frac{g}{z_{c}} y-\frac{k_{x}}{z_{c}}(x \ddot{y}-\ddot{x} y)-\frac{1}{m z_{c}} u_{r}, \\
& \ddot{x}=\frac{g}{z_{c}} x+\frac{k_{y}}{z_{c}}(x \ddot{y}-\ddot{x} y)+\frac{1}{m z_{c}} u_{p},
\end{aligned}
$$

where $u_{r}, u_{p}$ are new virtual inputs which are introduced to compensate input nonlinearity,

$$
\begin{aligned}
& u_{r}=\frac{D}{C_{r}} \tau_{r}, \\
& u_{p}=\frac{D}{C_{p}} \tau_{p},
\end{aligned}
$$

In the case of the walking on a flat plane, we can set the horizontal constraint plane $\left(k_{x}=0, k_{y}=0\right)$ and we obtain

$$
\begin{aligned}
& \ddot{y}=\frac{g}{z_{c}} y-\frac{1}{m z_{c}} u_{r}, \\
& \ddot{x}=\frac{g}{z_{c}} x+\frac{1}{m z_{c}} u_{p} .
\end{aligned}
$$

In the case of the walking on a slope or stairs where $k_{x}, k_{y} \neq 0$, we need another constraint. From $x \times(5)-y \times(6)$ we obtain

$$
x \ddot{y}-\ddot{x} y=\frac{-1}{m z}\left(u_{r} x+u_{p} y\right) .
$$

Therefore, we have the same dynamics of Eq. (9) and Eq. (10) in the case of an inclined constraint plane when the following new constraint is introduced about the inputs as,

$$
u_{r} x+u_{p} y=0 .
$$

This implies that the ankle torques should be given in a manner not generating the yaw moment so that the $Z$ element of angular momentum is conserved.

Eqs. (9) and (10) are independent linear equations. The only parameter which governs those dynamics is $z_{c}$, i.e., the $\mathrm{z}$ intersection of the constraint plane and the inclination of the plane never affects the horizontal motions. Note that the original dynamics were nonlinear and we have derived linear dynamics without using any approximation.

Let us call this the Three-Dimensional Linear Inverted Pendulum Mode (3D-LIPM). The first author 
and Tani introduced a two-dimensional version of this dynamics mode[6], and Hara, Yokokawa and Sadao extended it to three dimensions in the case of zero input torque[2].

\subsection{Nature of the 3D Linear Inverted Pendulum Mode}

We examine a nature of trajectories under the 3DLIPM with zero input torques $\left(u_{r}=u_{p}=0\right)$.

$$
\begin{aligned}
\ddot{y} & =\frac{g}{z_{c}} y \\
\ddot{x} & =\frac{g}{z_{c}} x .
\end{aligned}
$$

With a given initial condition, these equations determine trajectories in 3D space. Figure 2 shows two examples.

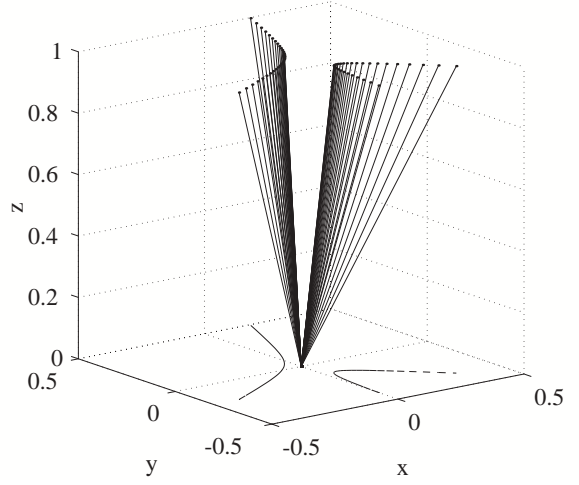

Figure 2: 3D Linear Inverted Pendulum Mode

Eqs. (13) and (14) can be regarded as a repulsive force field for a unit mass.

$$
f=\frac{g}{z_{c}} r
$$

A unit mass gets a force of magnitude $f$ which is proportional to the distance $r$ between the mass and the origin. Therefore, the 3D-LIPM with zero input torque can be considered as a dynamics under the central force field.

Figure 3 shows a 3D-LIPM trajectory which is projected onto $X Y$ plane. Motion along $Y$ and $X$ is governed by Eqs. (13) and (14) respectively. In Fig.3, it is also shown another coordinate frame $X^{\prime} Y^{\prime}$ which rotates $\theta$ from the original frame $X Y$. Since the 3DLIPM is a dynamics under the central force field, the motion along $Y^{\prime}$ and $X^{\prime}$ is also governed by the identical equations with Eqs. (13) and (14). This gives us great advantages in generating walking pattern as we will see in the following sections.

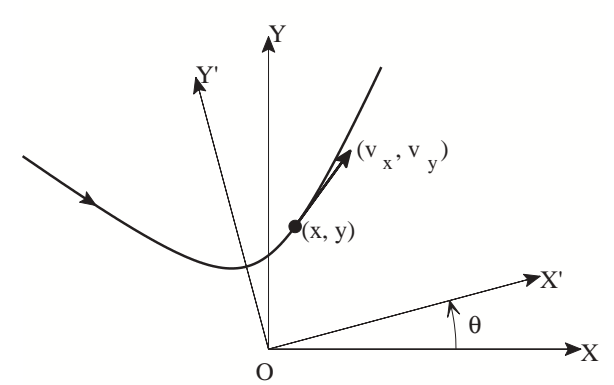

Figure 3: 3D-LIPM projected onto XY plane

\section{$3 \quad 3 \mathrm{D}$ walking pattern generation}

\subsection{Outline}

Figure 4 shows an example of a walking pattern based on the 3D-LIPM. In this paper we assume the support leg exchanges at a constant pace. To change the walking speed and direction, the robot modifies its foot placements (shown as small circles in Fig. 4).

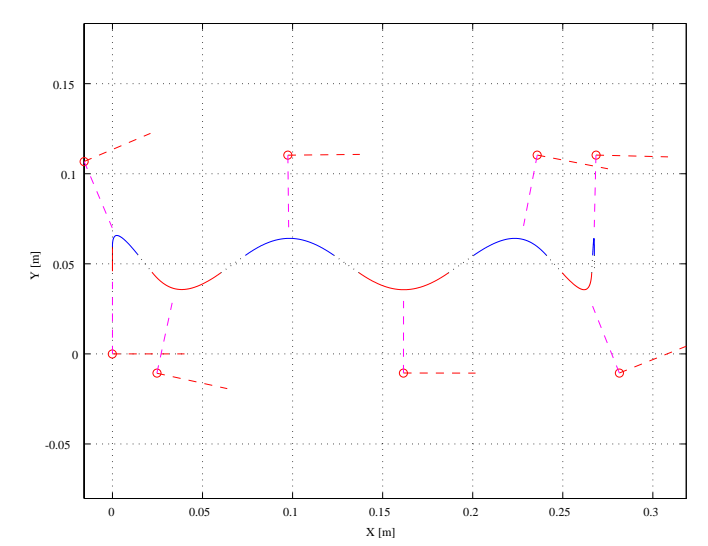

Figure 4: Walking pattern generated from the 3DLIPM. A robot takes seven steps from left to right. Motion of the tip of the inverted pendulum is shown as pieces of hyperbolic curves (solid lines). We assume that the robot is in double support and moves on a straight line between each support phase (dotted lines). Small circles are foot places and dashed lines indicate primary axes of the hyperbolic curves.

When we project the walking motion onto $X$ and $Y$ axis, we observe decoupled motions governed by Eqs. (13) and (14) (Fig. 5). Each motion follows the 2D version of the Linear Inverted Pendulum Mode that we described in the former paper [6]. 

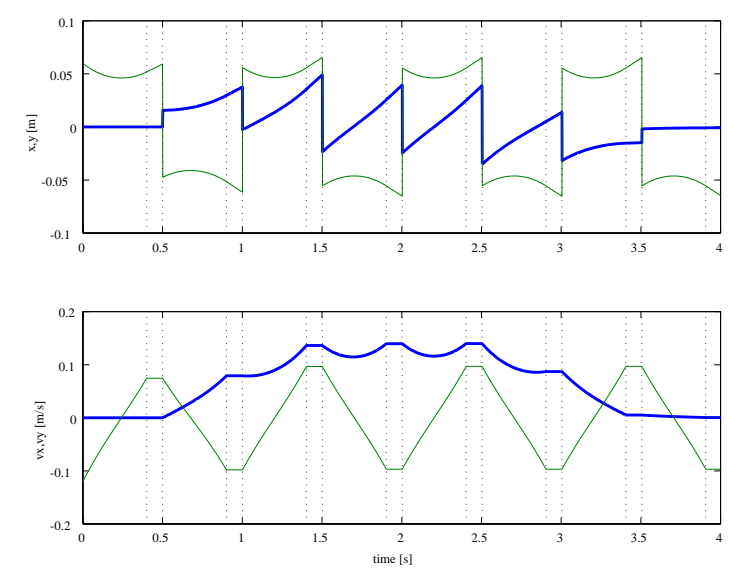

Figure 5: XY-position and velocity in a walk of the figure 4. The thick line shows $x$ motion and the thin line shows y motion. The position graphs jump the distance of the step length at each support foot exchange, since we are taking the origin at a foot place of each support. The vertical dotted lines indicate the time of the supporting mode change. In this walking pattern, the robot is taking 0.4 [sec] for single support and 0.1 [sec] for double support.

\subsection{Pattern generation along a local axis}

Now the problem becomes a control of the motion along $X$ or $Y$-axis for each step. Let us assume that the robot is repeating single support phase of duration $T_{s}$ and double support phase of duration $T_{d b l}$.

Figure 6 illustrates successive steps in $X$-direction. The initial body state $\left(x_{i}^{(n)}, v_{i}^{(n)}\right)$ and the final body state $\left(x_{f}^{(n)}, v_{f}^{(n)}\right)$ have the relationship given by

$$
\left(\begin{array}{c}
x_{f}^{(n)} \\
v_{f}^{(n)}
\end{array}\right)=\left(\begin{array}{cc}
C_{T} & T_{c} S_{T} \\
S_{T} / T_{c} & C_{T}
\end{array}\right)\left(\begin{array}{c}
x_{i}^{(n)} \\
v_{i}^{(n)}
\end{array}\right),
$$

where

$$
C_{T} \equiv \cosh \left(T_{s} / T_{c}\right), S_{T} \equiv \sinh \left(T_{s} / T_{c}\right), T_{c} \equiv \sqrt{z_{c} / g} .
$$

To control the walking speed, we must change the foothold (point E) to modify the initial condition of the support phase $\left(\mathrm{D}^{\prime} \rightarrow \mathrm{F}\right)$. When the desired status at the end of support (point $\mathrm{F}$ ) is given as $\left(x_{d}, v_{d}\right)$, we can define the norm of the error with certain weight $a, b>0$ as

$$
N \equiv a\left(x_{d}-x_{f}^{(2)}\right)^{2}+b\left(v_{d}-v_{f}^{(2)}\right)^{2} .
$$

By substituting Eq. (16) into this definition and calculating the foothold of $x_{i}^{(2)}$ which minimizes $N$, we obtain a proper control law,

$x_{i}^{(2)}=\left(a C_{T}\left(x_{d}-S_{T} T_{c} v_{d}\right)+b S_{T} / T_{c}\left(v_{d}-C_{T} v_{f}\right)\right) / D_{T}$,

where

$$
D_{T} \equiv a C_{T}^{2}+b\left(S_{T} / T_{c}\right)^{2} .
$$

To determine the foothold E, we also need the distance that body travels in the double support. The distance $d$ is given by

$$
d=v_{f}^{(1)} T_{d b l} .
$$

The motion of the swing leg is planned to arrive at the point $\mathrm{E}$ at the expected touchdown time (dashed curve from A to E in Fig. 6).

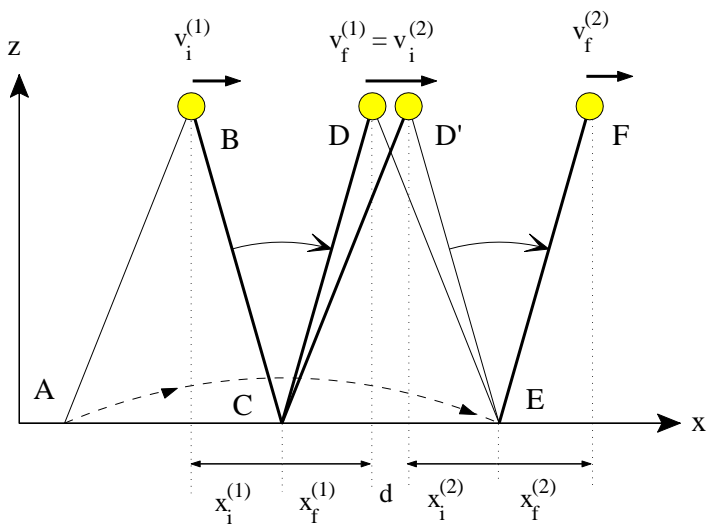

Figure 6: Two successive steps in the sagittal plane are illustrated. The body travels from $B$ to $D$ in the single-leg support phase, then moves from $D$ to $D$ ' in the double-leg support phase with constant speed $v_{f}^{(1)}$, and then travels $D$ ' to $F$ in the second single-support phase. While the body moves from $B$ to $D$, the tip of the swing leg travels from $A$ to $E$ (dashed curved line). By changing the position of $E$ we can control the final body speed $v_{f}^{(2)}$ at the point F. Except for our inserted double-support phase, this is the same idea proposed by Miura and Shimoyama [8].

\subsection{Control of walking direction}

To specify the walking direction, we rotates the reference $X Y$-frame at a foot place. Suppose the robot is walking along the $X$ axis and we want to change the walking direction with $\theta$ at $P_{3}$ (Figure 7). For this purpose, we define the $X^{\prime} Y^{\prime}$-frame which rotates $\theta$ during the support foot is at $P_{3}$ and use this new 


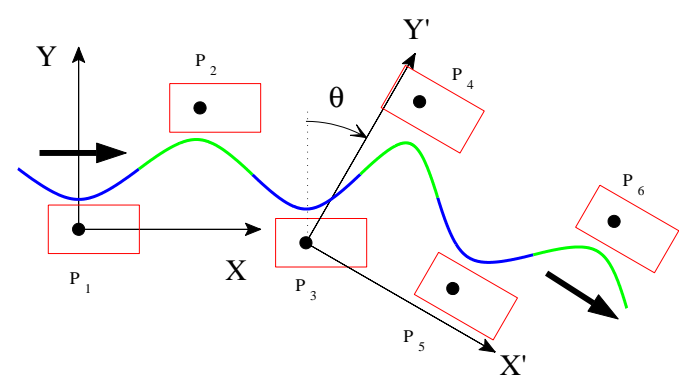

Figure 7: Changing walking direction

frame for the calculations after that. First, current body position and speed are converted, then the new foot place $P_{4}$ is calculated from the desired walking speed given in the new frame. This simple method works well even the frame rotations occures at every steps. Figure 8 illustrates a walking along a circle. In this walking pattern, the reference frame is rotated $\pi / 10 \mathrm{rad}$ so that the robot returns the stating point with 20 steps.

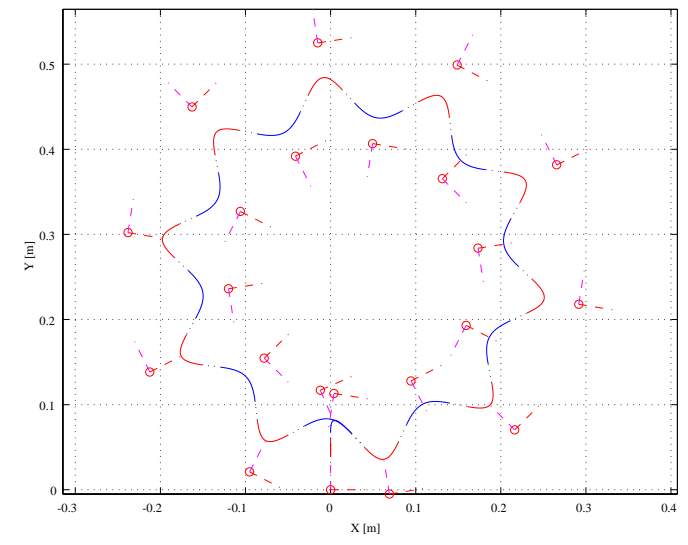

Figure 8: Walk on a circle

\section{Experiments of realtime bipped walking}

\subsection{Biped robot HRP-2L}

The biped robot HRP-2L, which is the leg module for HRP- $2^{1}$, is used for the experiments. Figure 9

\footnotetext{
${ }^{1}$ HRP-2 is a new humanoid robot platform, whose manufacturing process is in progress in phase two of Humanoid Robotics
}

shows the appearance of HRP-2L.
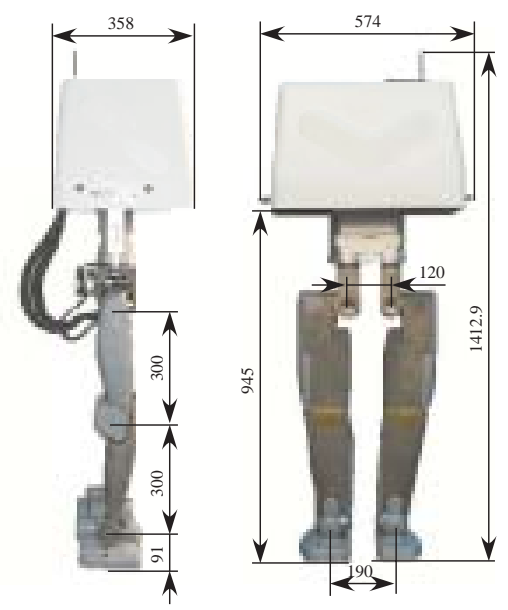

Figure 9: Biped robot HRP-2L

Its weight is $58.2[\mathrm{~kg}]$ including $11.4[\mathrm{~kg}]$ batteries and $22.6[\mathrm{~kg}]$ dummy weights corresponding to those of arms and head of HRP-2 under development. Each leg has 6 d.o.f. It has an onboard PC (Pentium III 933 $[\mathrm{MHz}])$ in the body. The operating system is ARTLinux[16].

\subsection{Control system of HRP-2L}

Recentrly, the walking of Asimo from Honda and H7 from the University of Tokyo have been succeessfully controled by a joystick in realtime $[4,11]$. We also developed a motion control software based on the proposed method for realizing realtime control of the walking motion and for interfacing an input device as shown in Fig. 10. The function of each module is described below.

Input Device Server runs on another PC. It gets current status of the input devices such as joystick and game pad. Foothold and rotation of the reference frame shown in Fig. 3 in the next step are calculated.

Walk Generator is the implemetation of the method described in this paper. It ganerates the desired pose of both legs and ZMP according to a command from the input device server. It runs at $200[\mathrm{~Hz}]$ cycle.

Stabilizer is a sensor feedback module that stabilizes the walking motion. In order to reduce the body

Project (HRP) of Ministry of Economy, Trade and Industry (METI) of Japan. 


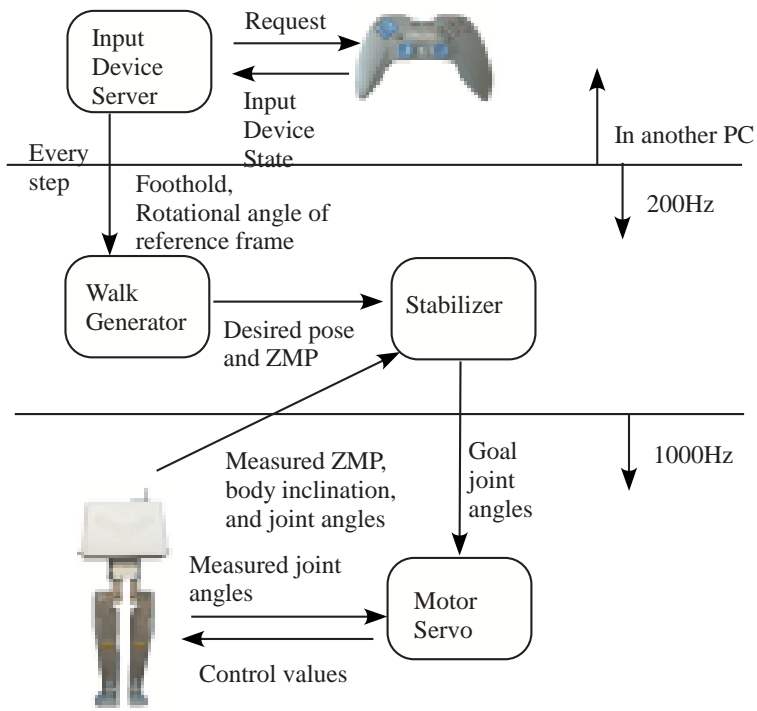

Figure 10: Control system for realtime generation of biped walking and input device interface

inclinations, each foot's desired position and orientation are adjusted as an inverted pendulum. In order to reduce the error between the desired ZMP trajectory and the actual ZMP, the horizontal position of the torso is adjusted. Finally the goal angles are calculated by solving the corresponding inverse kinematics. It also runs at 200 $[\mathrm{Hz}]$ cycle.

Motor Servo is a software PD servo module with $1000[\mathrm{~Hz}]$ cycle.

\subsection{Experimental results}

In order to evaluate the ability of the proposed realtime biped walk generation, experiments controlling HRP-2L by a game pad were performed. In the experiments, we select a flat floor so that the body height was kept constant. One more point that we must consider is the body and the foot rotation around $z$-axis. Although we assume an ideal robot, which can step towards any direction at all time, in the former section, HRP-2L has the limit of joint angles and it must avoid collision between the left and the right legs. For this reason, we designed additional pattern for the foot orientation with respect to the body, so that the body faces instantaneous walking direction in the middle of each support.

Figure 11 shows the snapshots of the experiment and Fig. 12 shows the body trajectory as the thick lines, foot placements as the small circles, and the measured ZMP trajectory as the thin lines. HRP-2L took nineteen steps in the experiment. It went forward at first 3 steps, turned right from 4th step to 11th step, then went backward to the end of steps. The time for each step was constant at 0.8 [sec]. However the distance between the two foot placements and the walking direction changed from $-0.25[\mathrm{~m}]$ to +0.25 $[\mathrm{m}]$ and from 0 [rad] to $0.34[\mathrm{rad}]$ in real time, respectively. From the experimental results, the effectiveness of the proposed realtime walk generation method was confirmed.

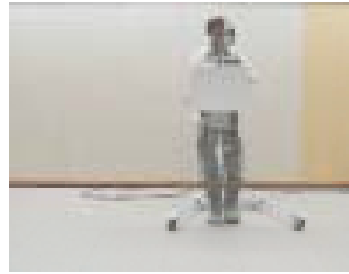

1st step

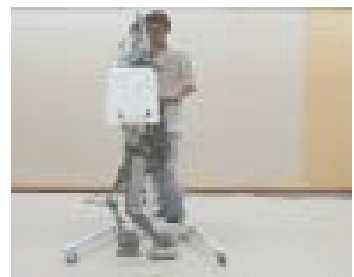

8th step

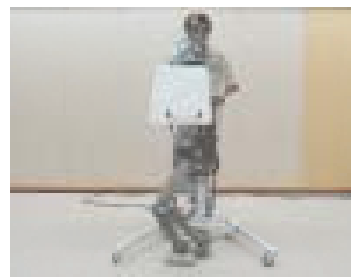

15 th step

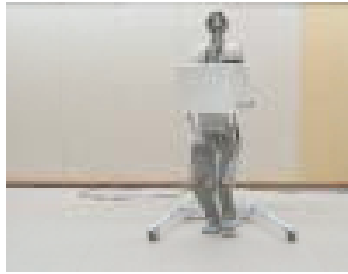

4th step

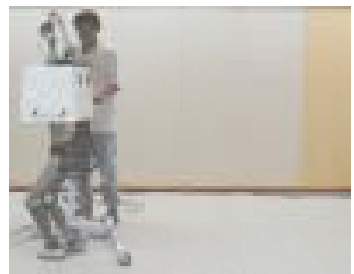

12th step

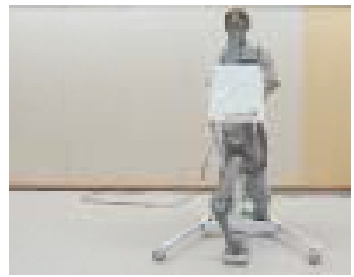

19th step
Figure 11: Snapshots of experiment

\section{Conclusions}

In this paper, we introduced the Three-Dimensional Linear Inverted Pendulum Mode (3D-LIPM) that is useful for realtime walking control in a $3 \mathrm{D}$ space. We discussed a nature of the 3D-LIPM and proposed a simple algorithm for walking pattern generation that can specify walking speed and direction. The realtime walk generation was tested on a 12 d.o.f. biped robot HRP-2L with the game pad interface, and a dynamically stable walk was successfully performed. 


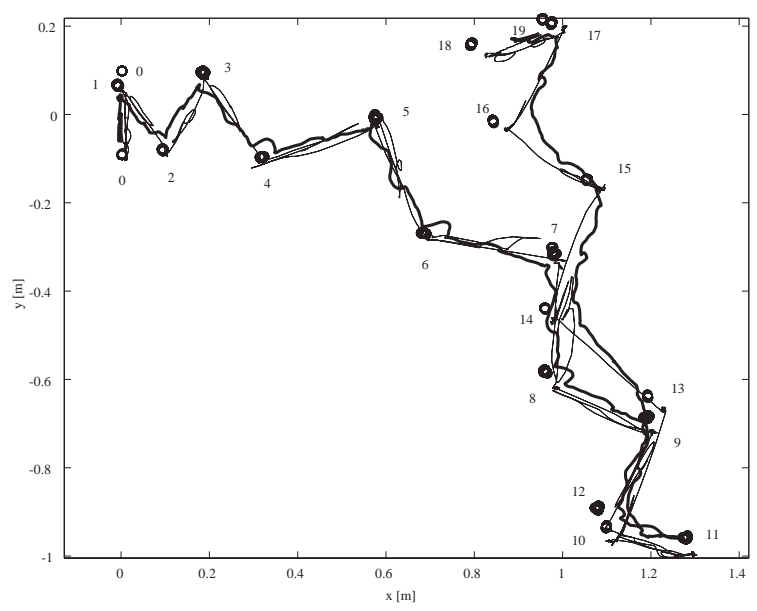

Figure 12: Hip motion, foot place, and ZMP

\section{Acknowledgments}

This research was supported by the Humanoid Robotics Project of the Ministry of Economy, Trade and Industry.

\section{References}

[1] Furusho, J. and Sano, A., "Sensor-based control of a nine-link biped," Int. J. Robotics Research, vol. 9, no. 2, pp. 83-98, Apr. 1990.

[2] Hara,K., Yokokawa,R. and Sadao, K., "Dynamic Control of Biped Locomotion Robot for Disturbance on Lateral Plane," Proc. of The Japan Society of Mechanical Engineers 72nd kansai meeting, pp. 10-37-10-38, 1997 (in Japanese).

[3] Hirai, K., Hirose,M., Haikawa, Y. and Takenaka, T., "The Development of Honda Humanoid Robot," Proc. IEEE Int. Conf. Robotics and Automations, pp. 1321-1326, 1998.

[4] Hirose, M., Haikawa, Y., and Takenaka, T., "Introduction of Honda Humanoid Robots Development," Proc. Advanced Science Institute 2001, No. 16, pp. 1-8, 2001.

[5] Huang, Q., Kajita, S. et.al, "A High Stability, Smooth Walking Pattern for a Biped Robot," Proc. IEEE Int. Conf. Robotics and Automations, pp. 65-71, 1999.

[6] Kajita,S. and Tani, K., "Study of Dynamic Biped Locomotion on Rugged Terrain," Proc. IEEE Int.
Conf. Robotics and Automations, pp. 1405-1410, 1991.

[7] Kajita,S., Matsumoto,O. and Saigo, M., "Realtime 3D walking pattern generation for a biped robot with telescopic legs," Proc. IEEE Int. Conf. Robotics and Automations, pp. 2299-2308, 2001.

[8] Miura,H. and Shimoyama, I., "Dynamic walk of a biped," International Journal of Robotics Research, Vol. 3, No. 2, pp. 60-72, 1984.

[9] Sano, A. and Furusho, J. , "Realization of Natural Dynamic Walking Using The Angular Momentum Information," Proc. IEEE Int. Conf. Robotics and Automations, pp. 1476-1481, 1990.

[10] Nishiwaki, K., Nagasaka, K., Inaba, M. and Inoue, H., "Generation of reactive stepping motion for a humanoid by dynamically stable mixture of pre-designed motions," Proc. IEEE Int. Conf. on Systems, Man, and Cybernetics, No. VI, pp. 702707, 1999.

[11] Nishiwaki, K., Sugihara, T., Kagami, S., Inaba, M., and Inoue, H., "Online Mixture and Connection of Basic Motions for Humanoid Walking Control by Footprint Specification," Proc. IEEE Int. Conf. Robotics and Automation, pp. 4110-4115, 2001.

[12] Pratt, J., Dilworth, P. and Pratt, G., "Virtual Model Control of a Bipedal Walking Robot," Proc. IEEE Int. Conf. Robotics and Automations, pp. 193-198, 1997.

[13] Raibert, M., Legged Robots that Balance, Cambridge, MA, MIT Press, 1986.

[14] Yamaguchi, J.,Soga,E., Inoue, S. and Takanishi, A., "Development of a Bipedal Humanoid Robot Control Method of Whole Body Cooperative Dynamic Biped Walking -," Proc. IEEE Int. Conf. Robotics and Automations, pp. 368-374, 1999.

[15] Zheng, Y.F. and Shen, J., "Gait Synthesis for the SD-2 Biped Robot to Climb Sloping Surface," IEEE Trans. Robotics and Automation, vol.6, no.1, pp. 86-96, 1990.

[16] Ishiwata, Y. and Matsui, T. "Development of Linux which has Advanced Real-Time Processing Function," Proc. RSJ Annual Conf., pp. 355-356, 1998. 